Dirk Roggenbuck*, Severine Vermeire, Ilse Hoffman, Dirk Reinhold, Peter Schierack, Alexander Goihl, Ulrike von Arnim, Gert De Hertogh, Dimitrios Polymeros, Dimitrios P. Bogdanos ${ }^{a}$ and Xavier Bossuyta

\title{
Evidence of Crohn's disease-related anti-glycoprotein 2 antibodies in patients with celiac disease
}

DOI 10.1515/cclm-2014-0238

Received March 4, 2014; accepted October 3, 2014; previously published online November 20, 2014

\section{Abstract \\ Background: Autoantibodies to exocrine-pancreatic gly- coprotein 2 (anti-GP2) are Crohn's disease (CD) markers. However, CD-specific antibodies have also been found in celiac-disease $(\mathrm{CeD})$ patients, in which type 1 diabetes- specific autoantibodies against endocrine pancreatic targets can be present. We investigated whether anti-GP2}

aDimitrios P. Bogdanos and Xavier Bossuyt share senior authorship. *Corresponding author: Prof. Dr. Dirk Roggenbuck, MD PhD, Faculty of Natural Sciences, Brandenburg Technical University Cottbus-Senftenberg, Grossenhainer Str. 57, 01968 Senftenberg, Germany, Phone: +49 33708 441716, Fax: +49 33708 441725, E-mail: dirk.roggenbuck@hs-lausitz.de; and Medipan GmbH, Dahlewitz/Berlin, Germany

Severine Vermeire: Department of Internal Medicine, Gastroenterology, University Hospitals Leuven, Catholic University of Leuven, Leuven, Belgium

Ilse Hoffman: Department of Pediatrics, University Hospitals Leuven, Catholic University of Leuven, Leuven, Belgium Dirk Reinhold and Alexander Goihl: Institute of Molecular and Clinical Immunology, Otto-von-Guericke University, Magdeburg, Germany

Peter Schierack: Faculty of Sciences, Brandenburg Technical University Cottbus-Senftenberg, Senftenberg, Germany

Ulrike von Arnim: Department of Gastroenterology, Hepatology and Infectious Diseases, Otto-von-Guericke University, Magdeburg, Germany

Gert De Hertogh: KU Leuven Department of Imaging and Pathology, University Hospitals Leuven, Leuven, Belgium

Dimitrios Polymeros: Hepatogastroenterology Unit, Department of Internal Medicine, Attikon Hospital, University of Athens, Athens, Greece

Dimitrios P. Bogdanos: Division of Transplantation Immunology and Mucosal Biology, King's College London School of Medicine at King's College Hospital, London, UK

Xavier Bossuyt: KU Leuven Department of Microbiology and Immunology, Catholic University Leuven and Department of Laboratory Medicine, Immunology, University Hospitals Leuven, Leuven, Belgium are also present in $\mathrm{CeD}$, a disease like $\mathrm{CD}$ which is also characterised by intestinal mucosal inflammation with barrier impairment.

Methods: Antibodies against GP2, tissue transglutaminase (tTG), deamidated gliadin (dGD), glutamic decarboxylase (GAD), and islet antigen-2 (IA2) were tested in sera from 73 CD patients, 90 blood donors (BD), and 79 (58 de novo) CeD patients (2 consecutive sera were available from 40 patients).

Results: IgA and/or IgG anti-GP2 were found in 15/79 (19.0\%) CeD patients on at least one occasion, in 25/73 (34.2\%) CD patients, and in 4/90 (4.4\%) BD (CeD vs. CD, $\mathrm{p}=0.042 ; \mathrm{BD}$ vs. $\mathrm{CeD}$ and $\mathrm{CD}, \mathrm{p}<0.001$, respectively). Amongst the 58 de novo CeD patients, anti-GP2 IgA and/ or IgG were present in $11(19.0 \%)$. Anti-GP2 IgA was significantly less prevalent in CeD compared with $\mathrm{CD}(\mathrm{p}=0.004)$. Anti-GP2 IgA and IgG in CD patients demonstrated a significantly higher median level compared to patients with $\mathrm{CeD}$ ( $\mathrm{p}<0.001, \mathrm{p}=0.008$, respectively). IgA anti-GP2 levels correlated significantly with IgA anti-tTG and anti-dGD levels in CeD Spearman's coefficient of rank correlation $(\rho)=0.42$, confidence interval $(\mathrm{CI}): 0.26-0.56, \mathrm{p}<0.001$; $\rho=0.54$, CI $0.39-0.65, p<0.001$, respectively.

Conclusions: The presence of anti-GP2 in CeD patients supports the notion that loss of tolerance to GP2 can probably be a manifestation of an autoinflammatory process in this intestinal disorder.

Keywords: celiac disease; Crohn's disease; glycoprotein GP2; intestinal autoimmunity.

\section{Introduction}

Glycoprotein 2 (GP2) has been identified as the main autoantigenic target of Crohn's disease (CD)-specific pancreatic antibodies (PAB) [1-3]. Apart from its previously assumed restricted location in the pancreas, recent data have demonstrated that GP2 is also a constituent of 
microfold (M) cells of the follicle-associated epithelium, which appears to have an antimicrobial effect, like its renal homolog uromodulin (Tamm-Horsfall protein) [46]. Additionally, emerging evidence indicates that GP2 is over-expressed at the site of intestinal inflammation in patients with $\mathrm{CD}$, and that this molecule modulates innate and adaptive immune responses $[1,7,8]$. Loss of tolerance to GP2 has been reported in up to $30 \%$ of CD patients compared to approximately $8 \%-10 \%$ of patients with ulcerative colitis (UC), the other main inflammatory bowel disease (IBD) [9-11]. The clinical significance of these autoantibodies has been assessed and seropositivity for anti-GP2 antibodies appears to identify CD patients with ileocolonic location, stenosing behaviour, and early disease onset [6, 12-16].

Of interest to the present study, CD-related PAB have been detected in patients with celiac disease (CeD), a chronic small intestinal immune-mediated enteropathy precipitated by exposure to dietary gluten in genetically predisposed individuals [17, 18]. Exposure to gluten in these patients triggers inflammatory processes leading to a variable degree of intestinal damage which is reversible with the initiation of gluten-free diet (GFD) [18]. The destructive mucosal changes detected in duodenal and jejunal biopsies lead to villous atrophy with hyperplasia of the crypts, a raised intraepithelial lymphocyte count, and an impairment of the intestinal barrier, a clinical complication also seen in patients with IBD [19, 20]. In contrast to the mucosal inflammatory changes in $\mathrm{CeD}$, the transmural inflammation in CD covers all layers of the bowel wall and adventitia and can occur throughout the intestinal tract $[3,21]$. Severe tissue lesions, such as fissures, abscesses, strictures, and fistulas, can develop in the course of $\mathrm{CD}$ [3].

The immunopathogenesis of IBD as well as that of CeD are poorly understood [22-24]. Antigen-driven mechanisms of immunological breakdown operate in both conditions, but it is still unclear whether the loss of tolerance to GP2 can also be seen in a sub-group of patients with CeD.

In the present study, we considered that it could be of interest to assess $\mathrm{PAB}$-specific anti-GP2 antibody reactivity in patients with $\mathrm{CeD}$, especially in those with de novo manifested disease before initiation of GFD. To this end, we studied IgA and IgG anti-GP2 in well-defined cohorts of serum samples from patients with CeD collected at two different time points over the course of the disease. We then compared the evidence of GP2 autoreactivity in $\mathrm{CeD}$ with that in a large number of patients with CD and healthy blood donors. Finally, we assessed the magnitude of PAB-related anti-GP2 antibodies in comparison to that of antibodies against the endocrine pancreas, such as those against the type 1 diabetes (T1D)-specific glutamic acid decarboxylase
(GAD) and islet antigen-2 (IA2) autoantigens, as well as the CeD-related human tissue transglutaminase (tTG) autoantigen and deamidated gliadin (dGD).

\section{Materials and methods}

\section{Subjects}

Three cohorts of patients with CeD were enrolled in the study including: 1) 42 patients with $\mathrm{CeD}$ (including 21 with de novo $\mathrm{CeD}$ ) from the Department of Laboratory Medicine, University Hospitals Leuven, Catholic University of Leuven; 2) six patients with de novo CeD from the Department of Gastroenterology, Hepatology and Infectious Diseases, Otto-von-Guericke University, Magdeburg; and 3) 31 with de novo CeD from the Department of Internal Medicine, Attikon Hospital, University of Athens, respectively. Overall, 58 of the 79 (73.4\%) CeD patients had de novo manifested CeD. Demographics and laboratory correlates of the $\mathrm{CeD}$ patients including Marsh histopathological classification is given in Table 1.

Seventy-three patients with CD from Leuven Hospital and 90 control sera from healthy blood donors (BD) were also assessed. Clinical diagnoses were based upon standard clinical, radiological, endoscopic and histological criteria.

The study was approved by the Local Ethics Committee and complies with the World Medical Association Declaration of Helsinki regarding ethical conduct of research involving human subjects and/ or animals. Aliquots of the sera stored at $-20{ }^{\circ} \mathrm{C}$ were used to detect antibody reactivity.

\section{Detection of anti-GP2 antibodies by ELISA}

Anti-GP2 IgG and IgA antibodies were assessed in serum samples of patients and controls by a commercially available enzyme-linked immunosorbent assay (ELISA) (GA Generic Assays, Dahlewitz/Berlin, Germany), according to the manufacturer's instructions [9]. These assays employ recombinant human GP2 expressed in Spodoptera frugiperda 9 cells, as described elsewhere [9]. Optical density was read in a microplate reader at $450 \mathrm{~nm}$ and results expressed as arbitrary units $(\mathrm{U} / \mathrm{mL})$. The cut-off for positivity at $20 \mathrm{U} / \mathrm{mL}$ was used for both anti-GP2 IgA and IgG antibody evaluation in accordance with the recommendations of the manufacturer. The functional assay sensitivity representing the lowest antibody concentration with a coefficient of variation of smaller than $20 \%$ was determined at $2.4 \mathrm{U} / \mathrm{mL}$ and $1.8 \mathrm{U}$ /mL for anti-GP2 IgG and anti-GP2 IgA, respectively.

\section{ELISA for the detection of CeD-specific antibodies}

Serum IgA and IgG antibodies to human recombinant tTG (anti-tTG) and dDG (anti-dGD) were determined by ELISA according to the instructions of the manufacturer (GA Generic Assays $\mathrm{GmbH}$ ) [27]. The optical density was read in a microplate reader at $450 \mathrm{~nm}$ and results expressed as arbitrary units $(\mathrm{U} / \mathrm{mL})$. The cut-off for positivity at $10 \mathrm{U} / \mathrm{mL}$ in accordance with the recommendations of the manufacturer was used for these assays. The functional assay sensitivity 
Table 1 Patients' and blood donors' (BD) characteristics.

\begin{tabular}{lrrr}
\hline & CeD & CD & BD \\
\hline $\mathrm{n}$ & $79^{\mathrm{a}}$ & 73 & 90 \\
Mean age (IQR) & $24.0(12.0-42.0)$ & $36.5(30.0-43.0)$ & $29.5(22.0-41.8)$ \\
Gender f/m & $55 / 24$ & $38 / 35$ & $33 / 57$ \\
De novo disease & 58 & na \\
2nd sample & $40^{\mathrm{b}}$ & 0 & 0 \\
Marsh classification & & & \\
1 & 1 & & \\
2 & 1 & & \\
3a & 12 & 28 & \\
3b & 20 & 17 & \\
3c & 12 & \\
Not available & & \\
\hline
\end{tabular}

${ }^{a}$ Nine of 42 patients with celiac disease (CD) referred to Leuven Hospital were from other institutions. For all 9 patients, anti-tissue transglutaminase (tTG) antibody levels were $>10$ times the upper limit of normal, and for 8 of them anti-tTG antibody levels were $>13$ times the upper limit of normal (Genesis Diagnostics Ltd, Littelport, UK). The likelihood ratio for CeD is 117 and 319 for anti-tTG levels $>10$ times and $>13$ times the upper limit of normal, respectively [25]. Furthermore, all 9 patients demonstrated double positivity of antibodies to tTG and deamidated gliadin (dGD), which is highly indicative for CeD [26]. One of these patients had dermatitis herpetiformis and 1 patient had type 1 diabetes with transient elevation of anti-tTG as well as anti-dGD IgA and IgG (no biopsy data available and no GFD). ${ }^{\mathrm{b} T h e}$ median time delay between the first and second sample analyzed was 9 months (minimum 3 months, 25 percentile 5 months, 75 th percentile 22 months and maximum 66 months). One patient reintroduced gluten (with concomitant increase in IgA anti-tTG, whereas another patient did not follow a strict diet (with increases in IgA anti-tTG levels). CD, Crohn's disease; IQR, interquartile range; na, not available.

[28] was determined at $2 \mathrm{U} / \mathrm{mL}$ and $3 \mathrm{U} / \mathrm{mL}$ for anti-tTG and anti-dGD IgA, respectively, and $3 \mathrm{U} / \mathrm{mL}$ and $2 \mathrm{U} / \mathrm{mL}$ for IgG to tTG and dGD, respectively.

\section{ELISA for the detection of T1D-specific antibodies}

Anti-GAD (anti-GAD) and anti-IA2 antibodies (anti-IA2) were evaluated by ELISA according to the instructions of the manufacturer (Medipan GmbH, Dahlewitz/Berlin, Germany). Briefly, ELISA plates coated with recombinant GAD or IA2 (islet cell antigen 512) were incubated with neat serum samples over night at $2-8{ }^{\circ} \mathrm{C}$. The assay system employs the ability of anti-GAD and anti-IA2 antibodies acting bivalently and forming a bridge between the immobilised autoantigens and their liquid-phase biotinylated variants. The biotinylated autoantigens bound by the respective serum antibodies allow antibody quantification through interaction with streptavidin-peroxidase and the corresponding colorogenic substrate. Absorbance values (at $450 \mathrm{~nm}$ ) were expressed as international units (IU) calibrated against World Health Organization (WHO) reference preparation NIBSC 97/550 for anti-GAD and anti-IA2 antibodies, respectively.

The cut-offs for positivity at $5 \mathrm{IU} / \mathrm{mL}$ for anti-GAD and at $10 \mathrm{IU} /$ $\mathrm{mL}$ for anti-IA2 antibodies were used in accordance with the recommendations of the manufacturer. The functional assay sensitivity was determined as $4 \mathrm{IU} / \mathrm{mL}$ and $0.8 \mathrm{IU} / \mathrm{mL}$ for anti-GAD and anti-IA2 antibodies, respectively.

\section{Statistical analysis}

A Kolmogorov-Smirnov test was used to analyse the data for normality. The measured values were expressed as medians with $95 \%$ confidential intervals (CI). The two-tailed, non-parametric Mann-Whitney and Kruskal-Wallis tests were used to test for statistically significant differences of independent samples in two or more groups, respectively. The non-parametric Wilcoxon test was employed to test paired samples.

Spearman's rank correlation test was applied for within group comparison. Comparison of prevalence rates between groups was performed by two-tailed Fisher's exact test. p-Values of $<0.05$ were considered significant. Calculations were performed using Medcalc statistical software (Medcalc, Mariakerke, Belgium).

\section{Results}

\section{Prevalence of autoantibodies in gastrointestinal patients and controls}

Anti-GP2 IgG positivity was not significantly different in patients with CeD compared to CD patients $(9 / 79,11.4 \%$ vs. 8/73, 11.0\%; $\mathrm{p}>0.05$ ) whereas anti-GP2 IgA positivity was (6/79, 7.6\% vs. 19/73, 26.0\%; $\mathrm{p}=0.004)$ (Table 2). Overall, IgA and/or IgG anti-GP2 reactivity was present in 15/79 (19.0\%) patients with CeD, which was also significantly less prevalent compared to patients with $\mathrm{CD}(25 / 73,34.2 \%$, $\mathrm{p}=0.042$ ). Both $\mathrm{CeD}$ and $\mathrm{CD}$ patient groups demonstrated a significantly higher prevalence of IgA and/or IgG antiGP2 reactivity than $\mathrm{BD}(4 / 90,4.4 \% ; \mathrm{p}=0.003, \mathrm{p}<0.001$, respectively). Amongst the 58 de novo CeD, 11 (19.0\%) had IgA and/or IgG anti-GP2 responses, respectively. The prevalences of IgA and/or IgG anti-GP2 seropositivity did not 
Table 2 Prevalence of autoantibodies to glycoprotein 2 (anti-GP2), glutamic acid decarboxylase (anti-GAD), islet cell antigen 2 (anti-IA2), tissue transglutaminase (anti-tTG), and deamidated gliadin (anti-dGD) in 79 celiac disease (CeD) patients (at least once in 40 patients with two consecutive samples), 73 Crohn's disease (CD) patients, and 90 blood donors (BD) detected by commercial enzyme-linked immunosorbent assay (ELISA).

\begin{tabular}{|c|c|c|c|c|c|c|c|c|c|}
\hline & $\begin{array}{r}\text { IgG } \\
\text { anti-GP2, \% }\end{array}$ & $\begin{array}{r}\operatorname{IgA} \\
\text { anti-GP2, \% }\end{array}$ & $\begin{array}{r}\text { IgG/IgA } \\
\text { anti-GP2, \% }\end{array}$ & $\begin{array}{r}\text { Anti-GAD, } \\
\%\end{array}$ & $\begin{array}{r}\text { Anti-IA2, } \\
\%\end{array}$ & $\begin{array}{r}\text { IgA anti-tTG, } \\
\%\end{array}$ & $\begin{array}{r}\text { IgG anti-tTG, } \\
\%\end{array}$ & $\begin{array}{r}\text { IgA anti-dGD, } \\
\%\end{array}$ & $\begin{array}{r}\text { IgG anti-dGD, } \\
\%\end{array}$ \\
\hline $\mathrm{CeD} n=79^{\mathrm{a}}$ & $9(11.4)$ & $6(7.6)$ & $15(19.0)$ & $18(22.8)$ & $5(6.3)$ & $76(96.2)$ & $76(96.2)$ & $64(81.0)$ & 69 (87.3) \\
\hline$C D n=73$ & $8(11.0)$ & $19(26.0)$ & $25(34.2)$ & $0(0.0)$ & $1(1.4)$ & $0(0.0)$ & $4(5.5)$ & $0(0.0)$ & $1(1.4)$ \\
\hline $\mathrm{BD} n=90$ & $2(2.2)$ & $2(2.2)$ & $4(4.4)$ & $2(2.2)$ & $1(1.1)$ & nd & nd & nd & nd \\
\hline
\end{tabular}

differ between the two largest cohorts from Leuven and Athens of de novo CeD patients (5/21 vs. 4/31, $\mathrm{p}>0.05$ ).

As expected, CeD-specific antibodies such as IgA and IgG anti-tTG and anti-dGD demonstrated significantly higher prevalences in patients with $\mathrm{CeD}$ compared to those with $\mathrm{CD}$ (Table 2, $\mathrm{p}<0.001$, respectively).

\section{Concentration of autoantibodies in gastrointestinal patients and controls}

IgG and IgA anti-GP2 antibody levels differed significantly amongst patients with $\mathrm{CD}, \mathrm{CeD}$, and $\mathrm{BD}$ (Kruskal-Wallis test, $\mathrm{p}<0.001$; Figure 1). Anti-GP2 IgA in CD patients demonstrated a significantly higher median level compared to patients with $\mathrm{CeD}[8.0 \mathrm{U} / \mathrm{mL}$, interquartile range (IQR): 4.7-17.1 vs. 4.6 U/mL, IQR: 2.0-7.7; $p<0.001$ ]. There was also a significantly elevated concentration of anti-GP2 IgG antibodies in patients with CD compared to patients with CeD (8.0 U/mL, IQR: 5.3-11.3 vs. 5.6 U/mL, IQR: 2.5-10.2; $\mathrm{p}=0.008)$.

The concentration of anti-GAD in patients with $\mathrm{CD}$, CeD, and BD was also significantly different (Kruskal-Wallis test, $\mathrm{p}<0.001$; Figure $2 \mathrm{~A}$ ). In contrast to anti-GP2, anti-GAD levels were significantly higher in patients with $\mathrm{CeD}$ compared to those with $\mathrm{CD}(\mathrm{p}<0.001)$. Anti-IA2 also demonstrated different concentrations in the respective groups $(p<0.001$; Figure $2 B)$, whereas its level was significantly higher in patients with CeD compared with $\mathrm{CD}(\mathrm{p}=0.004)$.

In patients with $\mathrm{CeD}$, IgA anti-GP2 levels correlated significantly with IgA anti-tTG and anti-dGD levels [Spearman's coefficient of rank correlation $(\rho)=0.42$, CI $0.26-$ $0.56, p<0.001 ; \rho=0.54$, CI 0.39-0.65, $p<0.001$, respectively; Figure 3]. In contrast, anti-GP2 IgG did not correlate with IgG anti-tTG or with anti-dGD antibodies ( $\mathrm{p}>0.05$ ).

\section{CeD-, CD-, and T1D-specific antibodies in follow-up samples of $\mathrm{CeD}$ patients}

Anti-tTG IgA and IgG antibody levels as well as their corresponding prevalences were significantly higher in the first of the two CeD follow-up samples (Table 3, p<0.001, respectively). In particular, the median concentrations of anti-tTG IgA and IgG in the first and second samples were as follows: $143.4 \mathrm{U} / \mathrm{mL}, \mathrm{IQR}: 76.4-320.0$ vs. $8.8 \mathrm{U} / \mathrm{mL}$, IQR:
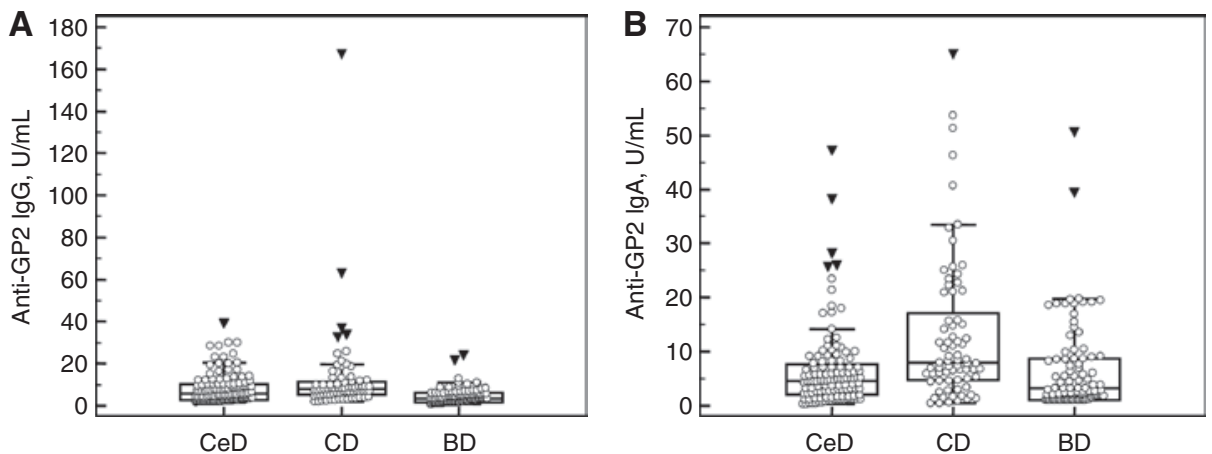

Figure 1 IgG and IgA to glycoprotein 2 (anti-GP2) in 119 samples of patients with celiac disease (CeD), 73 samples of patients with Crohn's disease (CD), and 90 blood donor samples (BD) detected by commercial enzyme-linked immunosorbent assay (ELISA).

Data are displayed in Box-and-Whisker plots with far out values, defined as values that are smaller than the lower quartile minus 3 times the interquartile range, or larger than the upper quartile plus 3 times the interquartile range, displayed as solid triangles. 

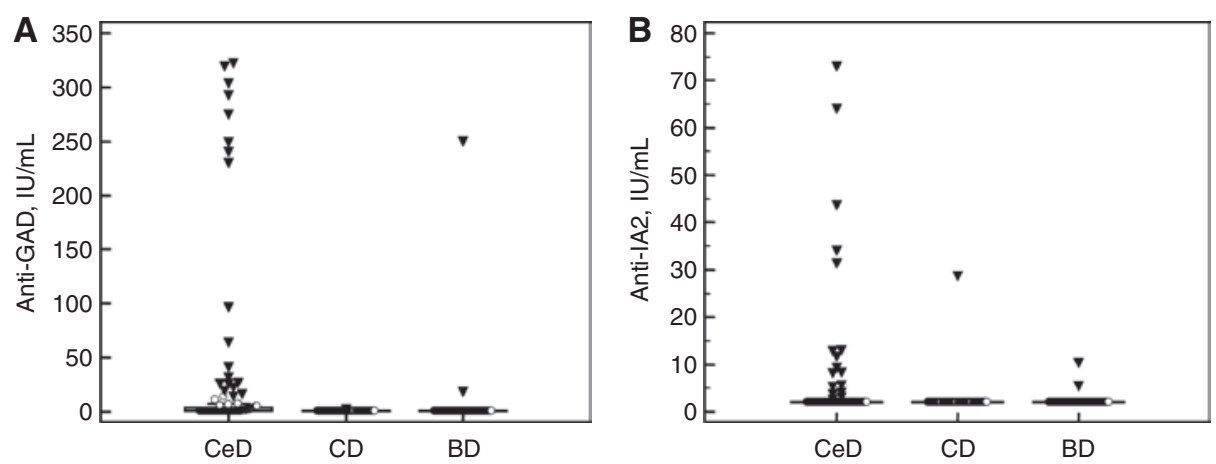

Figure 2 Autoantibodies to glutamic acid decarboxylase (anti-GAD) and insel cell antigen 2 (anti-IA2) in 119 samples from 79 patients with celiac disease (CeD), 73 samples of patients with Crohn's disease (CD), and 90 blood donor samples (BD) detected by commercial enzymelinked immunosorbent assay (ELISA).

Data are displayed in Box-and-Whisker plots with far out values, defined as values that are smaller than the lower quartile minus 3 times the interquartile range, or larger than the upper quartile plus 3 times the interquartile range, displayed as solid triangles.

5.5-28.8 and 79.7 U/mL, IQR: $37.0-201.4$ vs. $10.8 \mathrm{U} / \mathrm{mL}$, IQR: 5.5-30.3; respectively, Figure 4A, B). The median concentrations of anti-dGD IgA and IgG antibodies were also higher in the first compared to the second $\mathrm{CeD}$ sample $(\mathrm{p}<0.001$, respectively): Anti-dGD IgA were $17.7 \mathrm{U} / \mathrm{mL}$, IQR: $8.8-51.2$ vs. $5.3 \mathrm{U} / \mathrm{mL}$, IQR: $2.4-9.0$ and anti-dGD IgG were $31.7 \mathrm{U} / \mathrm{mL}$, IQR: 11.1-90.6 vs. $6.5 \mathrm{U} / \mathrm{mL}$, IQR: 3.1-20.2. Prevalences of anti-dGD IgA and IgG were also significantly higher in the first compared to the second sample of the $40 \mathrm{CeD}$ patients with a follow-up sample (Table 3 , $\mathrm{p}<0.001, \mathrm{p}=0.001$, respectively).

Although there was not a significantly different prevalence for anti-GP2 IgA and/or IgG in the follow-up samples, IgA anti-GP2 levels did show a significant reduction in the second sample (4.6 U/mL, IQR: $2.4-8.9$ vs. 2.7 $\mathrm{U} / \mathrm{mL}, \mathrm{IQR}: 1.1-5.1 ; \mathrm{p}<0.001)$. There was also a significantly diminished anti-GP2 IgG antibody reactivity in the second sample compared to the first (7.2 U/mL, IQR: $2.4-13.8$ vs.
4.7 U/mL, IQR: 1.7-7.0; $\mathrm{p}=0.033$ ) (Figure $4 \mathrm{C}, \mathrm{D}$ ). Interestingly, whereas the prevalence of anti-GP2 IgA and/or IgG was still not significantly different in the first samples of patients with $\mathrm{CeD}$ from that in patients with $\mathrm{CD}$, the 40 second samples of CeD patients, mainly under GFD, demonstrated a significant lower prevalence thereof $(3 / 40$, $8.5 \%$ vs. $25 / 73,34.2 \% ; p=0.001)$. Furthermore, the prevalence of anti-GP2 IgA and/or IgG in the second samples did not differ significantly from that in $\mathrm{BD}(\mathrm{p}>0.05)$, whereas the prevalence of these antibodies in the first samples did (8/40, 20.0\% vs. $4 / 90,4.4 \%, p=0.018)$.

In contrast, the median of anti-GAD and anti-IA2 levels as well as their corresponding prevalences were not different amongst follow-up samples ( $p>0.05$, respectively).

Amongst the $40 \mathrm{CeD}$ with follow-up serum samples, 37 (92.5\%) and 34 (85.0\%) have shown a decline of IgA and IgG anti-tTG antibodies in the second sample, respectively (Figure 4). All of the three CeD patients with rising
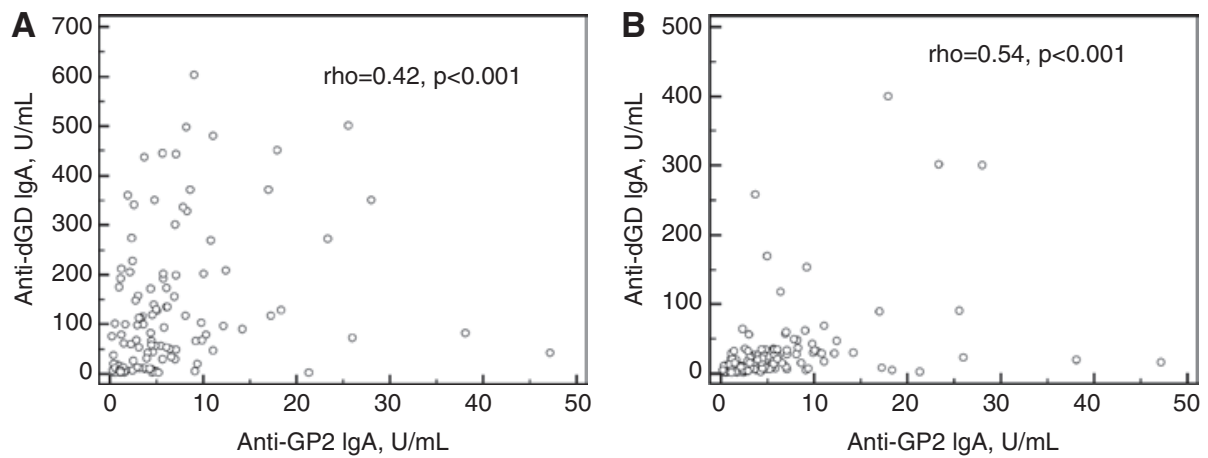

Figure 3 Correlation of anti-glycoprotein 2 (GP2) IgA with anti-tissue transglutaminase (tTG) and deamidated gliadin IgA concentrations detected by commercial enzyme-linked immunosorbent assay (ELISA) in 79 celiac disease patients ( 40 patients with two consecutive samples).

Spearman's coefficient of rank correlation $(\rho)=0.42, \rho=0.54 ; p<0.001$, respectively. 
Table 3 Prevalence of autoantibodies to glycoprotein 2 (anti-GP2), glutamic acid decarboxylase (anti-GAD), insel cell antigen 2 (anti-IA2), tissue transglutaminase (anti-tTG), and deamidated gliadin (anti-dGD) in 40 celiac disease (CeD) patients with two consecutive samples detected by commercial enzyme-linked immunosorbent assay (ELISA).

\begin{tabular}{|c|c|c|c|c|c|c|c|c|c|}
\hline $\operatorname{CeD} n=40$ & $\begin{array}{r}\text { IgG anti-GP2, } \\
\%\end{array}$ & $\begin{array}{r}\text { IgA anti-GP2, } \\
\%\end{array}$ & $\begin{array}{r}\operatorname{IgG} / \operatorname{IgA} \\
\text { anti-GP2, \% }\end{array}$ & $\begin{array}{r}\text { Anti-GAD, } \\
\%\end{array}$ & Anti-IA2, \% & $\begin{array}{r}\text { IgA anti-tTG, } \\
\%\end{array}$ & $\begin{array}{r}\text { IgG anti-tTG, } \\
\%\end{array}$ & $\begin{array}{r}\text { IgA anti-dGD, } \\
\%\end{array}$ & $\begin{array}{r}\text { IgG anti-dGD, } \\
\%\end{array}$ \\
\hline 1st samples & $6(15.0)$ & $2(5.0)$ & $8(20.0)$ & $8(20.0)$ & $3(7.5)$ & $38(95.0)$ & $38(95.0)$ & $27(67.5)$ & $31(77.5)$ \\
\hline 2nd samples & $2(5.0)$ & $1(2.5)$ & $3(7.5)$ & $9(22.5)$ & $3(7.5)$ & $17(42.5)$ & $21(52.5)$ & $7(17.5)$ & $16(40.0)$ \\
\hline
\end{tabular}

IgA anti-tTG levels demonstrated an increase of more than $20 \%$ compared to the first value. Of those three, one patient suffered from T1D and had CeD since 2 years under a GFD. The rising CeD-specific antibodies in this patient were due to the introduction of gluten into the diet. The second CeD patient of this group developed CeD and did not follow a strict GFD. The third CeD patient presented with de novo CeD.

\section{Discussion}

Both CD and CeD demonstrate inflammation of the intestinal mucosa. However, the localisation of the intestinal destruction and the pathophysiological mechanisms responsible for the induction of these diseases are quite distinct $[3,29]$. Nevertheless, the inflammatory processes seen in $\mathrm{CD}$ and $\mathrm{CeD}$ are believed to be exacerbated by or lead to an impairment of the intestinal barrier [30, 31]. Growing evidence has been collected to demonstrate that both clinical entities involve the loss of humoral tolerance to self and microbiota antigens [24, 32-36]. We demonstrated in the past, that loss of tolerance to GP2 antigen, an intestinal glycoprotein found apart from its pancreatic localisation in the intestinal $\mathrm{M}$ cells of the follicle-associated epithelium, is a characteristic feature of intestinal destruction in patients with $\mathrm{CD}[6,12,15,37]$. In agreement with previous studies reporting GP2 autoantibody detection in up to $30 \%$ of patients with CD, we found antiGP2 IgG and/or IgA antibody reactivity in 34.2\% patients with $\mathrm{CD}$. In contrast to the published data, the number
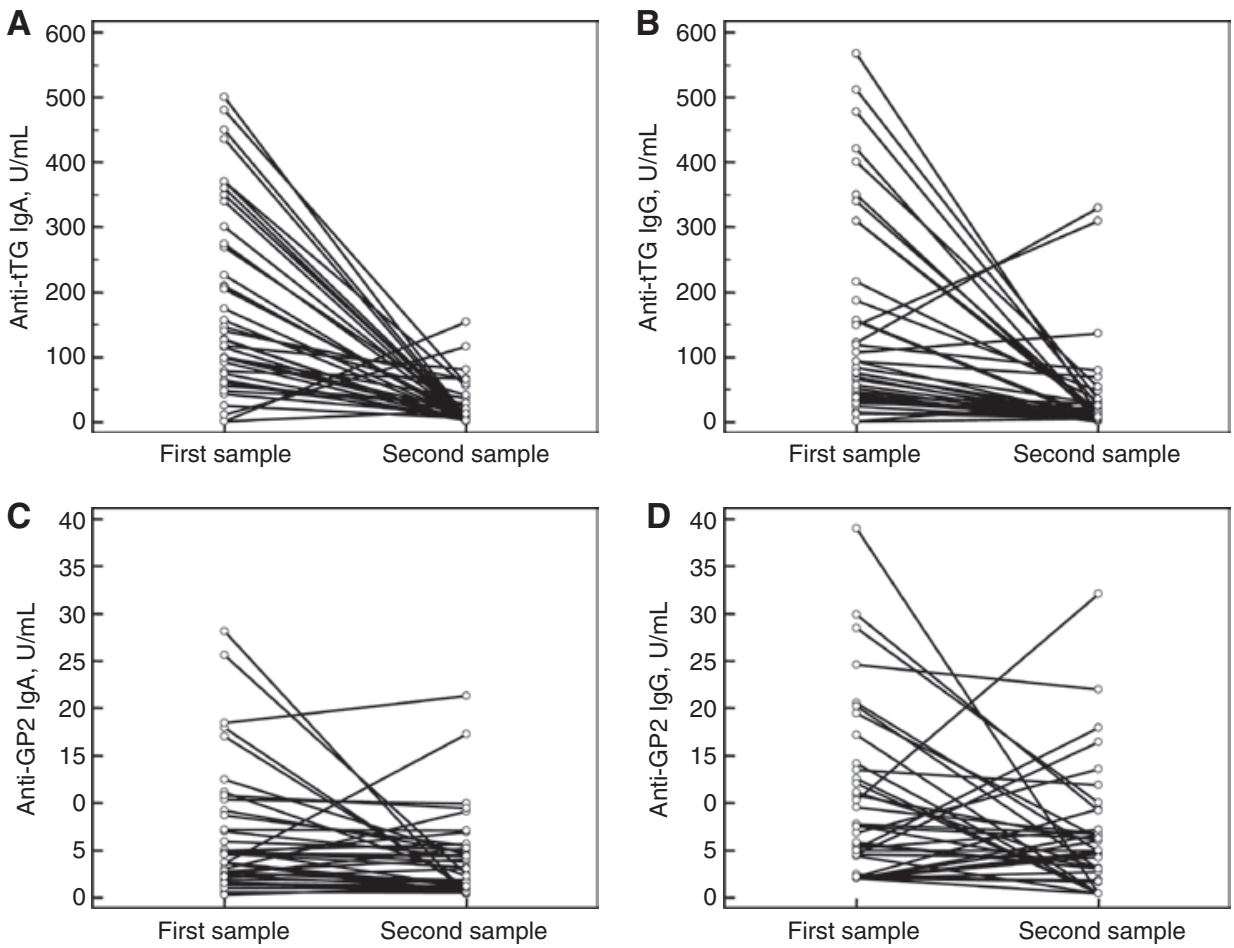

Figure 4 IgA and IgG anti-tissue transglutaminase (tTG) and anti-glycoprotein 2 (GP2) levels detected by commercial enzyme-linked immunosorbent assay (ELISA) in 40 celiac disease patients with two consecutive samples. 
of anti-GP2 IgA positive patients with CD in our series exceeded that of anti-GP2 IgG antibodies [9, 11, 38, 39]. The great majority of our CeD patients (60 out of 62 ) with a known histopathological assessment had a Marsh scoring of 3 preventing us from further analysis associating the presence of anti-GP2 antibodies with tissue destruction. This issue could be solved in a larger cohort including various Marsh scores.

We postulated that loss of tolerance to the GP2 specifically interacting with the intestinal microbiota [40] may be a generic feature of conditions characterised by an inflammation of the intestinal mucosa, and we assessed this hypothesis by testing patients with CeD. To some extent, our assumption has been a valid one. We have identified patients with CeD, in whom anti-GP2 antibodies were detectable. Our data were obtained testing three different patient cohorts, as well as a significant number of de novo manifested CeD using the same ELISA kits. Thus, we have been able not only to assess the prevalences of anti-GP2 antibodies in de novo $\mathrm{CeD}$ versus follow-up serum samples but also to compare GP2-specific responses amongst geographically distinct cohorts from Germany, Belgium and Greece. Our data reached a consensus: IgA anti-GP2 antibodies are present in a considerable number of CeD patients.

The frequency of anti-GP2 IgG seropositivity was not significantly different in the CeD compared to the $\mathrm{CD}$ cohort but was higher compared to that in BD. However, the median levels of IgA as well as IgG anti-GP2 were higher in CD patients. This phenomenon could be due to the lower amount of follicle-associated epithelium and, therefore, $\mathrm{M}$ cells in the duodenum, the typical site of inflammation during $\mathrm{CeD}$, compared with the ileum being one of the main inflammatory sites in CD [41-43]. We could support this assumption by revealing a significantly higher prevalence of anti-GP2 IgA and/or IgG in the first follow-up samples of patients with $\mathrm{CeD}$ containing patients with de novo $\mathrm{CeD}$ in contrast to that in $\mathrm{BD}$. The second samples of those patients with CeD mainly under GFD did not show this significant difference.

During the preparation of this manuscript, limited data in the form of a letter to the editor have also been presented suggesting that GP2 is an autoantibody target in some patients with $\mathrm{CeD}$. Bonaci-Nikolic and colleagues reported the occurrence of anti-GP2 in untreated de novo patients with $\mathrm{CeD}$, which points to the possibility of autoreactivity to GP2 during acute CeD inflammation characterised by a complete loss of the architecture of the small bowel in severe cases [17]. Further evidence of loss of tolerance to GP2 in CeD was reported recently demonstrating in particular anti-GP2 IgA in patients with refractory CeD [44].
We measured anti-GAD and anti-IA2 as internal autoantibody controls since these autoantibodies target T1D-related antigens located in the endocrine pancreas while GP2 is an autoantigen of pancreatic autoantibodies against the exocrine pancreas. In contrast to anti-GP2, anti-GAD and anti-IA2 seropositivity in patients with IBD was not significantly different to that in BD.

Interestingly, we could establish a significant correlation between IgA anti-GP2 and IgA anti-tTG as well as anti-dGD levels investigating 119 sera from the 79 patients with CeD. Also, it seems that autoreactivity to GP2 in CeD follows the trend of loss of tolerance to tTG but such an association could not be found for anti-GAD or anti-IA2. After the onset of a GFD, the CeD-typical inflammation largely disappears as did the antibody reactivity to tTG in the majority of clinical cases. Provided that the loss of tolerance to GP2 antigen in CeD behaves in a similar manner to the well-known autoreactivity to $\mathrm{TTG}$, anti-GP2 antibodies may be an element of intestinal inflammation rather than a sole hallmark of $\mathrm{CD}$. Whether removal of the putative causative inflammatory agent in $\mathrm{CD}$ could lead to a reduction of anti-GP2 levels as does removal of gluten for anti-tTG in $\mathrm{CeD}$ remains to be seen.

Recently, the occurrence of anti-GP2 has been reported in UC patients with an ileal pouch anal anastomosis developing pouchitis after proctocolectomy $[45,46]$. Interestingly, such patients with CeD-like complications demonstrated significantly higher anti-GP2 levels than those without. Noteworthy, the association of anti-GP2 levels with disease activity in $\mathrm{CD}$ has been discussed, albeit controversially. Clinical remission is not always accompanied by the disappearance of anti-GP2 [11, 47, 48].

Our data show a correlation between IgA anti-tTG and anti-dDG with anti-GP2 IgA. Whether this is a characteristic feature in $\mathrm{CeD}$ remains to be confirmed by further studies. Such correlations were not found in patients with $\mathrm{CD}$. The interpretation of these data has to be treated with extreme caution.

In summary, anti-GP2 IgG and IgA can occur during intestinal inflammation in a proportion of patients with $\mathrm{CeD}$. The diagnostic and clinical significance of these findings require external validation and further investigation in a large cohort of prospectively collected serum samples from patients with $\mathrm{CeD}$ followed up for a long time.

Acknowledgments: We thank Doreen Dillaerts for expert technical assistance.

Author contributions: All the authors have accepted responsibility for the entire content of this submitted manuscript and approved submission.

Financial support: None declared. 
Employment or leadership: Dirk Roggenbuck is a shareholder of GA Generic Assays GmbH and Medipan GmbH. The remaining authors declare that they have no competing financial interests.

Honorarium: None declared.

Competing interests: The funding organisation(s) played no role in the study design; in the collection, analysis, and interpretation of data; in the writing of the report; or in the decision to submit the report for publication.

\section{References}

1. Roggenbuck D, Hausdorf G, Martinez-Gamboa L, Reinhold D, Buttner T, Jungblut PR, et al. Identification of GP2, the major zymogen granule membrane glycoprotein, as the autoantigen of pancreatic antibodies in Crohn's disease. Gut 2009;58:1620-8.

2. Komorowski L, Teegen B, Probst C, Aulinger-Stocker K, Sina C, Fellermann K, et al. Autoantibodies against exocrine pancreas in Crohn's disease are directed against two antigens: the glycoproteins CUZD1 and GP2. J Crohns Colitis 2013;7:780-90.

3. Laass MW, Roggenbuck D, Conrad K. Diagnosis and classification of Crohn's disease. Autoimmun Rev 2014;13:467-71.

4. Hase K, Kawano K, Nochi T, Pontes GS, Fukuda S, Ebisawa M, et al. Uptake through glycoprotein 2 of FimH(+) bacteria by $M$ cells initiates mucosal immune response. Nature 2009;462:226-30.

5. Terahara K, Yoshida M, Igarashi O, Nochi T, Pontes GS, Hase K, et al. Comprehensive gene expression profiling of Peyer's patch $M$ cells, villous $M$-like cells, and intestinal epithelial cells. J Immunol 2008;180:7840-6.

6. Roggenbuck D, Reinhold D, Werner L, Schierack P, Bogdanos DP, Conrad K. Glycoprotein 2 antibodies in Crohn's disease. Adv Clin Chem 2013;60:187-208.

7. Werner L, Paclik D, Fritz C, Reinhold D, Roggenbuck D, Sturm A. Identification of pancreatic Glycoprotein 2 as an endogenous immunomodulator of innate and adaptive immune responses. J Immunol 2012;189:2774-83.

8. Holzl MA, Hofer J, Kovarik JJ, Roggenbuck D, Reinhold D, Goihl A, et al. The zymogen granule protein 2 (GP2) binds to scavenger receptor expressed on endothelial cells I (SREC-I). Cell Immunol 2011;267:88-93.

9. Roggenbuck D, Reinhold D, Wex T, Goihl A, von Arnim U, Malfertheiner P, et al. Autoantibodies to GP2, the major zymogen granule membrane glycoprotein, are new markers in Crohn's disease. Clin Chim Acta 2011;412:718-24.

10. Bogdanos DP, Rigopoulou El, Smyk DS, Roggenbuck D, Reinhold D, Forbes A, et al. Diagnostic value, clinical utility and pathogenic significance of reactivity to the molecular targets of Crohn's disease specific-pancreatic autoantibodies. Autoimmun Rev 2011;11:143-8.

11. Op de Beeck K, Vermeire S, Rutgeerts P, Bossuyt X. Antibodies to GP2, the major zymogen granule membrane glycoprotein, in inflammatory bowel diseases. Gut 2012;61:162-4.

12. Bogdanos DP, Roggenbuck D, Reinhold D, Wex T, Pavlidis $P$, von Arnim U, et al. Pancreatic-specific autoantibodies to glycoprotein 2 mirror disease location and behaviour in younger patients with Crohn's disease. BMC Gastroenterol 2012;12:102.
13. Pavlidis P, Romanidou O, Roggenbuck D, Mytilinaiou M, Al-Sult$\tan \mathrm{F}$, Liaskos C, et al. Ileal inflammation may trigger the development of GP2-specific pancreatic autoantibodies in patients with Crohn's disease. Clin Dev Immunol 2012;2012:640835.

14. Somma V, Ababneh H, Ababneh A, Gatti S, Romagnoli V, Bendia E, et al. The novel Crohn's disease marker anti-GP2 antibody is associated with ileocolonic location of disease. Gastroenterol Res Pract 2013;2013:683824.

15. Rieder F, Franke A, Dirmeier A, Lopez R, Lang S, Roggenbuck D, et al. Mo1247 serologic anti-GP2 antibodies are associated with strictures and need for surgical resection in Crohn's disease. Gastroenterology 2013;144:S617.

16. Roggenbuck D, Humbel RL, Reinhold D, Bogdanos DP, Conrad K, Laass MW. Glycoprotein 2 antibodies in inflammatory bowel disease - no association with disease phenotype? J Pediatr Gastroenterol Nutr 2012;56:e5.

17. Bonaci-Nikolic B, Spuran M, Andrejevic S, Nikolic M. Autoantibodies to GP2, the major zymogen granule membrane glycoprotein, in patients with gluten-sensitive enteropathy: a possible serological trap. Clin Chim Acta 2012;413:822-3.

18. Ludvigsson JF, Leffler DA, Bai JC, Biagi F, Fasano A, Green PH, et al. The Oslo definitions for coeliac disease and related terms. Gut 2013:62:43-52.

19. Soderholm JD, Olaison G, Lindberg E, Hannestad U, Vindels A, Tysk C, et al. Different intestinal permeability patterns in relatives and spouses of patients with Crohn's disease: an inherited defect in mucosal defence? Gut 1999;44:96-100.

20. Bjarnason I. Intestinal permeability. Gut 1994;35:S18-22.

21. Baumgart DC, Carding SR. Inflammatory bowel disease: cause and immunobiology. Lancet 2007;369:1627-40.

22. Rieder F, Lawrance IC, Leite A, Sans M. Predictors of fibrostenotic Crohn's disease. Inflamm Bowel Dis 2011;17:2000-7.

23. Bardella MT, Elli L, De MS, Floriani I, Torri V, Piodi L. Autoimmune disorders in patients affected by celiac sprue and inflammatory bowel disease. Ann Med 2009;41:139-43.

24. Basso D, Zambon CF, Plebani M. Inflammatory bowel diseases: from pathogenesis to laboratory testing. Clin Chem Lab Med 2014;52:471-81.

25. Vermeersch P, Geboes K, Marien G, Hoffman I, Hiele M, Bossuyt X. Diagnostic performance of IgG anti-deamidated gliadin peptide antibody assays is comparable to IgA anti-tTG in celiac disease. Clin Chim Acta 2010;411:931-5.

26. Vermeersch P, Geboes K, Marien G, Hoffman I, Hiele M, Bossuyt X. Serological diagnosis of celiac disease: comparative analysis of different strategies. Clin Chim Acta 2012;413:1761-7.

27. Conrad K, Roggenbuck D, Ittenson A, Reinhold D, Buettner T, Laass MW. A new dot immunoassay for simultaneous detection of celiac specific antibodies and IgA-deficiency. Clin Chem Lab Med 2011;50:337-43.

28. Zöphel K, Wunderlich G, Kotzerke J, von Landenberg P, Roggenbuck D. M22 based (manual) ELISA for TSH-receptor antibody (TRAb) measurement is more sensitive than 2nd generation TRAb assays. Clin Chim Acta 2009;403:266.

29. Sollid LM. Coeliac disease: dissecting a complex inflammatory disorder. Nat Rev Immunol 2002;2:647-55.

30. Tibble J, Sigthorsson G, Foster R, Sherwood R, Fagerhol M, Bjarnason I. Faecal calprotectin and faecal occult blood tests in the diagnosis of colorectal carcinoma and adenoma. Gut 2001;49:402-8.

31. de Kort S, Keszthelyi D, Masclee AA. Leaky gut and diabetes mellitus: what is the link? Obes Rev 2011;12:449-58. 
32. Bossuyt X. Serologic markers in inflammatory bowel disease. Clin Chem 2006;52:171-81.

33. Bonifacio E, Lampasona V, Genovese S, Ferrari M, Bosi E. Identification of protein tyrosine phosphatase-like IA2 (islet cell antigen 512) as the insulin-dependent diabetes-related $37 / 40 \mathrm{~K}$ autoantigen and a target of islet-cell antibodies. J Immunol 1995;155:5419-26.

34. Baekkeskov S, Kanaani J, Jaume JC, Kash S. Does GAD have a unique role in triggering IDDM? J Autoimmun 2000;15:279-86.

35. Conrad K, Schmechta H, Klafki A, Lobeck G, Uhlig HH, Gerdi S, et al. Serological differentiation of inflammatory bowel diseases. Eur J Gastroenterol Hepatol 2002;14:129-35.

36. Michielan A, Basso D, Martinato M, Pathak S, Banerjee A, Oliva L, et al. Increased antibody response to microbial antigens in patients with Crohn's disease and their unaffected first-degree relatives. Dig Liver Dis 2013;45:894-8.

37. Roggenbuck D, Reinhold D, Schierack P, Bogdanos DP, Conrad K, Laass MW. Crohn's disease specific pancreatic antibodies: clinical and pathophysiological challenges. Clin Chem Lab Med 2014;52:483-94.

38. Pavlidis P, Forbes A, Bogdanos DP. Antibodies to glycoprotein 2 (GP2) in patients with inflammatory bowel diseases from UK. Clin Chim Acta 2011;412:1163-4.

39. Roggenbuck D, Bogdanos D, Conrad K. Loss of tolerance to one or two major targets in Crohn's disease or just cross-reactivity? J Crohns Colitis 2013; 7:e273-4.

40. Schierack P, Rödiger S, Kolenda R, Hiemann R, Berger E, Grzymailo K, et al. Species-specific and pathotype-specific binding of bacteria to zymogen granule membrane glycoprotein 2 (GP2). Gut [Epub ahead of print 2014 Jul 29]. doi:10.1136/ gutjnl-2014-307854 (in press).

41. Colombel JF, Watson AJ, Neurath MF. The 10 remaining mysteries of inflammatory bowel disease. Gut 2008;57:429-33.

42. Gullberg E, Soderholm JD. Peyer's patches and M cells as potential sites of the inflammatory onset in Crohn's disease. Ann N Y Acad Sci 2006;1072:218-32.

43. Cornes JS. Peyer's patches in the human gut. Proc R Soc Med 1965;58:716.

44. Gross S, Bakker SF, van Bodegraven AA, van Hoogstraten IM, Gelderman KA. Increased IgA glycoprotein-2 specific antibody titres in refractory CD. J Gastrointestin Liver Dis 2014;23:1-7.

45. Werner L, Sturm A, Roggenbuck D, Yahav L, Zion T, Meirowithz E, et al. Antibodies against glycoprotein 2 are novel markers of intestinal inflammation in patients with an ileal pouch. J Crohns Colitis 2013;7:e522-32.

46. Werner L, Roggenbuck D, Dotan I. Reply to Dr. Pavlidis et al.'s letter. J Crohns Colitis 2013;7:e604-5.

47. Roggenbuck D, Reinhold D, Wex T, von Arnim U, Malfertheiner P, Sturm A, et al. Authors' response: antibodies to GP2, the major zymogen granule membrane glycoprotein, are specific for Crohn's disease and may reflect treatment response. Gut 2012;61:164-5.

48. Kovacs M, Lakatos PL, Papp M, Jacobsen S, Nemes E, Polgar M, et al. Pancreatic autoantibodies and autoantibodies against goblet cells in pediatric patients with inflammatory bowel disease (IBD). J Pediatr Gastroenterol Nutr 2012;55:429-35. 